\title{
Extra cardiac findings by 64-multidetector computed tomography in patients with symptomatic atrial fibrillation prior to pulmonal vein isolation
}

\author{
Christian Sohns · Samuel Sossalla • Dirk Vollmann • \\ Lars Luethje $\cdot$ Joachim Seegers $\cdot$ Jan D. Schmitto \\ Markus Zabel $\cdot$ Silvia Obenauer
}

Received: 29 September 2009/ Accepted: 3 June 2010/Published online: 13 June 2010

(C) The Author(s) 2010. This article is published with open access at Springerlink.com

\begin{abstract}
The aim of this study was to investigate the prevalence of extracardiac findings diagnosed by 64-multidetector computed tomography (MDCT) examinations prior to circumferential pulmonary vein (PV) ablation of atrial fibrillation (AF). A total of 158 patients (median age, 60.5 years; male 68\%) underwent 64-MDCT of the chest and upper abdomen to characterize left atrial and $\mathrm{PV}$ anatomy prior to $\mathrm{AF}$ ablation. MDCT images were evaluated by a thoracic radiologist and a cardiologist. For additional scan interpretation, bone, lung, and soft tissue window settings were used. CT scans with extra-cardiac abnormalities categorized for the anatomic distribution and divided into two groups: Group 1-exhibiting clinically significant or potentially significant findings, and Group 2-patients with clinically non-
\end{abstract}

Markus Zabel and Silvia Obenauer contributed equally.

C. Sohns $(\bowtie) \cdot$ S. Sossalla $\cdot$ D. Vollmann .

L. Luethje · J. Seegers · M. Zabel

Department of Cardiology and Pneumology/Heart Center,

Georg-August-University, Robert-Koch-Str. 40,

37075 Göttingen, Germany

e-mail: christian.sohns@gmx.de

\section{J. D. Schmitto}

Division of Cardiac Surgery, Brigham and Women's

Hospital, Harvard Medical School, Boston, MA, USA

S. Obenauer

Department of Radiology, Georg-August-University,

Göttingen, Germany significant findings. Extracardiac findings $(n=198)$ were observed in 113/158 (72\%) patients. At least one significant finding was noted in 49/158 patients (31\%). Group 1 abnormalities, such as malignancies or pneumonias, were found in $85 / 198$ findings (43\%). Group 2 findings, for example mild degenerative spine disease or pleural thickening, were observed in 113/ 198 findings (72\%). 74/198 Extracardiac findings were located in the lung (37\%), 35/198 in the mediastinum (18\%), 8/198 into the liver (4\%) and 81/198 were in other organs (41). There is an appreciable prevalence of prior undiagnosed extracardiac findings detected in patients with AF prior to PV-Isolation by MDCT. Clinically significant or potentially significant findings can be expected in $\sim 40 \%$ of patients who undergo cardiac MDCT. Interdisciplinary trained personnel is required to identify and interpret both cardiac and extra cardiac findings.

Keywords Extra cardiac findings - Atrial fibrillation $\cdot 64-M D C T \cdot$ Pulmonary vein ablation

\section{Introduction}

The current generation of multidetector CT (MDCT) scanners with submillimeter slice collimation and high temporal resolution permits robust, fast and reliable contrast-enhanced imaging of the coronary arteries, cardiac anatomy and great vessels [1-3]. Several studies have suggested that multidetector coronary 
CT angiography can accurately exclude significant coronary artery stenosis, with negative predictive values of $97-100 \%$, in comparison with invasive selective coronary angiography $[1,2]$. Coronary calcifications can also be quantitatively assessed with MDCT, allowing for a non-invasive measure of coronary atherosclerosis. The coronary artery calcium score quantifies coronary plaque burden and has been shown to be a predictor of cardiac events, allowing risk stratification in individual patients [3-5].

Another application of cardiac MDCT is to detail the left atrium and pulmonary veins before AF catheter ablation. Since Haissaguerre et al. [6] first described the elimination of arrhythmia triggers at the ostia of pulmonary veins by means of catheter based ablation, a very large number of ablation procedures have been performed worldwide [7]. As a consequence, the technology for AF ablation continues to evolve with a major focus being the incorporation of anatomical data into advanced three dimensional mapping and navigational systems. These systems are of high importance to the procedure as $40 \%$ of patients will have an anatomical variant [8]. The utility of MDCT assessment of the PV prior to ablation has been described and has contributed to high procedural success rates $[9,10]$. MDCT can provide pivotal information about the size, location, and number of pulmonary vein ostia.

In addition to the information about the anatomy of the heart, coronary arteries and great vessels, these examinations also include portions of the lung, mediastinum, chest wall, spine and upper abdomen. Some of the latter findings may account for patients symptoms, whereas other incidental findings may indicate underlying malignant disease or even remain indeterminate without further investigations or follow up. The aim of this study was to determine the prevalence and significance of extra cardiac findings in patients with symptomatic atrial fibrillation.

\section{Methods}

Patients. In a consecutive series, at the Heart Center of the University hospital of Goettingen (Germany), between April 2006 and January 2009, 158 patients (32\% female, $68 \%$ male) with symptomatic AF refractory to anti-arrhythmic medication underwent contrast-enhanced 64-MDCT of the chest and upper abdomen for identification of pulmonal vein anatomy before pulmonal vein isolation. Patient demographics were recorded for identification of the computer images and for later access of clinical information in the hospital database and files. The median age of the study group was 60.5 years (range 21-100 years). All patients were referred from the Division of Clinical Electrophysiology Center, in order to assist with pulmonary vein isolation in patients with symptomatic atrial fibrillation. Written informed consent was obtained from each patient.

Imaging. All patients were examined with a MDCT-scanner (VCT Light Speed, General Electric) which acquires 64 slices per gantry rotation at a gantry rotation time of $500 \mathrm{~ms}$. Patients were urged to hyperventilate before the CT-scan by taking several large breaths in and out to maximize breath-holding for the scan. The imaging is performed within a single breath-hold. Scan volume included the supraaortic region to the heart base and the upper abdomen. $80 \mathrm{ml}$ of intravenous contrast medium (Imeron 400, Bracco Imaging, Germany) was applied in all cases. For scanning in the craniocaudal direction, collimation of $64 \times 0.625 \mathrm{~mm}$ was used at a rotation time of $0.8 \mathrm{~s}$. Detector scan area was $40 \mathrm{~mm}$. Thickness was $0.625 \mathrm{~mm}$, tube voltage was $140 \mathrm{kV}$. Slice thickness of the reconstruction was $0.625 \mathrm{~mm}$. Axial slices were reconstructed using electrocardiographic-gated half scan reconstruction algorithm. Numerous phases were reconstructed within the cardiac cycle to optimize structure visualization without motion artifacts.

Image analysis. All images were displayed using an Advantage Windows Workstation (Volume Viewer 4.2, General Electric) and were evaluated by an attending thoracic radiologist and an attending cardiologist. For additional scan interpretation bone, lung, and soft tissue window settings were used. For each patient, the anatomy of the pulmonary vein ostia was identified. In addition, the following CT findings were described if present: pattern of pulmonary parenchymal abnormalities (nodules, air-space opacities, ground-glass attenuation, emphysema), mediastinal lymph node enlargement, aortic aneurysm, pleural effusion, diaphragmatic hernia, degenerative spine disease. Abnormalities in upper abdominal organs such as the liver and spleen were also described if visualized on the CT scan. Abnormalities and the respective patients were classified into two groups 
based on their estimated clinical relevance. Group 1 comprised patients with clinically significant or potentially significant findings requiring further workup, and Group 2 comprised patients with clinically nonsignificant findings who did not require any additional examinations. A finding was classified as significant if any recommendation within the clinical report for additional research such as imaging or clinical correlation was recommended. Group 1 included patients with nodules larger than $4 \mathrm{~mm}$, air-space opacity, ground-glass attenuation, moderate or severe emphysema, aortic aneurysm $(>4 \mathrm{~cm})$, enlarged mediastinal lymph nodes $(>10 \mathrm{~mm})$, pleural effusion, diaphragmatic hernia $(>2 \mathrm{~cm})$, and moderate or severe degenerative spine disease. Thus, this group exhibited for example malignancies and pneumonias. Group 2 comprised patients with small linear lung opacities, small $(<4 \mathrm{~mm})$ or calcified nodules, small mediastinal lymph nodes $(<10 \mathrm{~mm})$, and pleural thickening. An entirely normal CT scan result was also noted.

\section{Results}

At least one extracardiac finding was observed in $113 / 158(72 \%)$ of the patients. Overall, 198 extracardiac findings were noted. $31 \%$ of the patients had at least one significant or potentially significant finding. Clinically significant or potentially significant findings (Group 1) were found in 85 patients (43\%) and are summarized in Table 1. The most common findings in this group were mediastinal lymph nodes $>10 \mathrm{~mm}(9 \%)$ and moderate or severe degenerative spine disease (13\%; Table 1, Figs. 1, 2). Other significant findings included diaphragmatic hernia in nine patients, liver lesions in seven patients (Figs. 3, 4) and histological proven lung cancer in two patients (Fig. 5). Figure 1 shows the anatomic distribution of the extra cardiac findings for Group 1.

Non-significant clinical findings were found in $113 / 158(72 \%)$ of the patients. The most common findings in this group were mild degenerative spine disease (31.5\%; Fig. 3) and small and linear lung opacity ( $8.5 \%$; Table 2$)$. Other non-significant findings included mediastinal lymph nodes $<4 \mathrm{~mm}$ in 12 patients, aortic ectasis $<4 \mathrm{~mm}$ in 6 cases, or lipoma in one patient. The distribution of all findings of Group 2 is as well shown in Fig. 1.
Table 1 Distribution of clinical significant or potentially significant extra cardiac findings (Group 1)

\begin{tabular}{ll}
\hline Clinically significant or potentially significant & $\begin{array}{l}\text { Patients } \% /[n / \\
\text { CT findings }\end{array}$ \\
\hline Mediastinal lymph nodes $>10 \mathrm{~mm}$
\end{tabular}

\section{Discussion}

MDCT coronary angiography and coronary artery calcium scoring examinations are being carried out with increasing frequency as a non-invasive method for assessment of coronary atherosclerosis and for risk stratification in symptomatic and asymptomatic patients. Because AF is the most common cardiac arrhythmia, an increasing number of ablations are being performed at many centers. 3-D reformatted MDCT images of the left atrium and distal PVs provide this information, including the number, location and angulation of PVs and their ostial branches. Thus, MDCT imaging can act as a "road map" for the interventional cardiologist, as well as to provide a baseline for possible later complications, if they should appear $[9,10]$.

In the present study, the prevalence of extra cardiac findings by MDCT in patients with AF was found to be considerable. Since the field of view (FOV) in CT examinations includes several additional thoracic organs such as the mediastinal structures, the lung hila and parenchyma, the bony thoracic cage including the spine and the chest wall soft tissues, it is important to inspect all those structures for a potential abnormality. Not infrequently, chest pain or dyspnea 
A

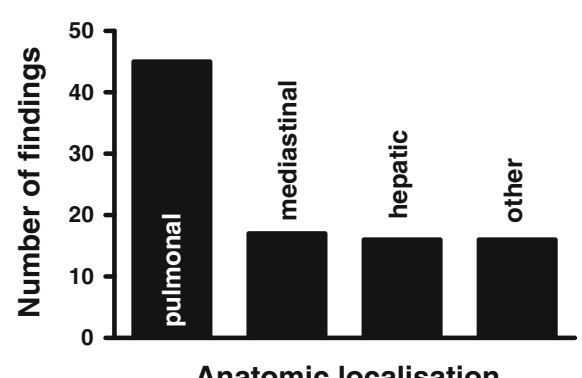

Anatomic localisation

Fig. 1 Anatomic distribution of all findings (Other localisations were for example: Spine, ribs, spleen, diaphragma). Group 1 comprised patients with clinically significant or
B

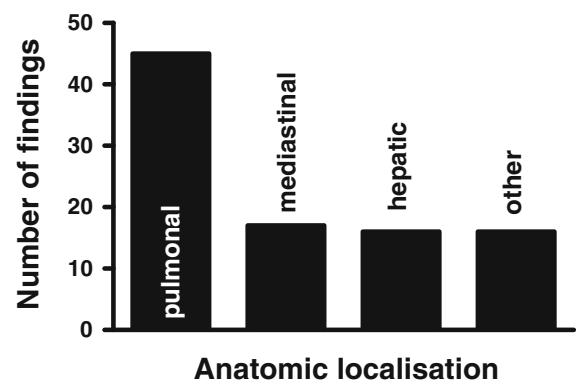

potentially significant findings requiring further workup, and Group 2 comprised patients with clinically non-significant findings
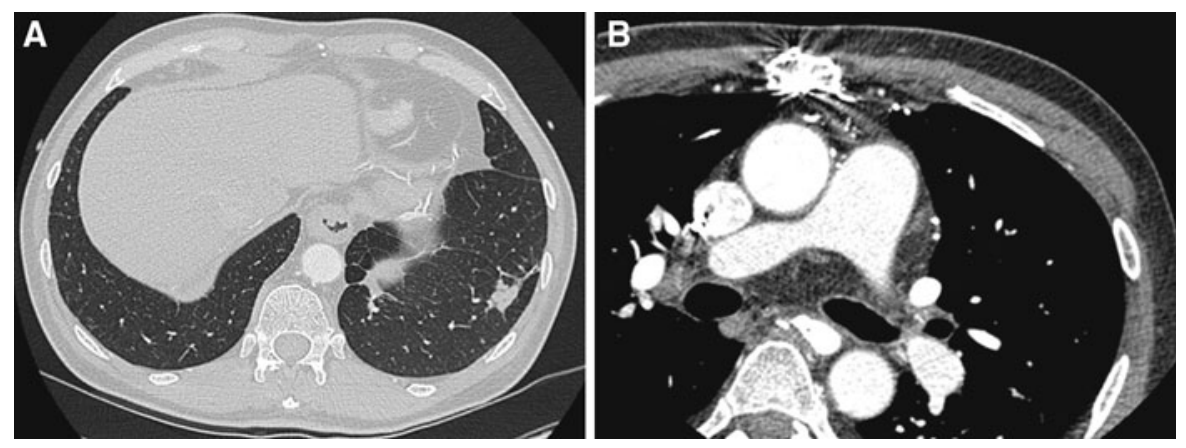

Fig. 2 58-year-old man with AF. a CT-slice at lung window with illustration of pulmonary nodules and infiltration on the left side. b CT-slice at mediastinal window showing mediastinal lymph nodes $>10 \mathrm{~mm}$

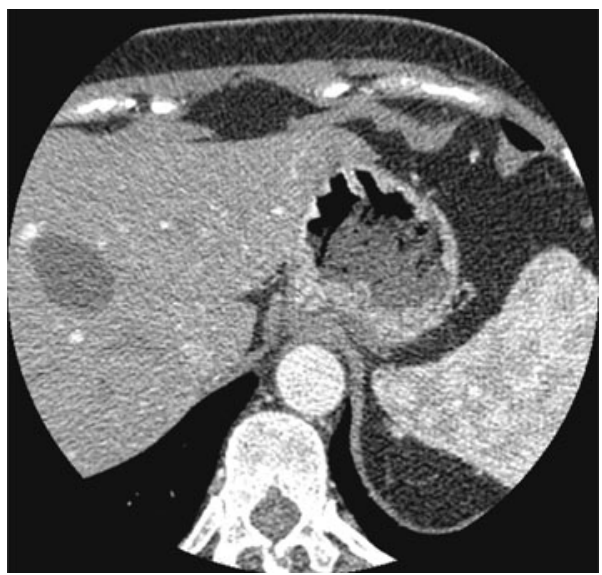

Fig. 3 61-year-old man with AF. CT-slice at standart window with demonstration of a cystic liver lesion

might be caused by disease of such extra cardiac organs. The prevalence of extra cardiac clinically significant findings in our study $(31 \%)$ is in agreement with other previous studies (Table 1, Fig. 1) [1-16].
In four large Electronic beam CT (EBCT) studies, the reported prevalence of incidental findings were $7.8 \%$ [11], 53\% [12], $8 \%$ [17], and 20.5\% [18] with the higher prevalence in the due to the inclusion of "other cardiovascular findings" which accounted for $61 \%$ of all incidental findings. Thus, the prevalence of extracardiac findings in these studies are estimated to be equal to our results (Tables 1, 2). Hunold et al. performed contrast-enhanced noninvasive coronary angiography in addition to coronary calcium EBCT in $32 \%$ of their studies. The additional findings diagnosed in their examination accounted $33 \%$ of all pulmonary findings and $45 \%$ of all pulmonary tumor [12]. In addition, 53\% of their study subjects had extracoronary pathological findings [12]. However, a large number of minor abnormalities was included, such as pulmonary scarring, atelectasis, degenerative arthritis and rib fractures, which were partially not included in our examination. Furthermore, the results suggest that the addition of a contrast examination 

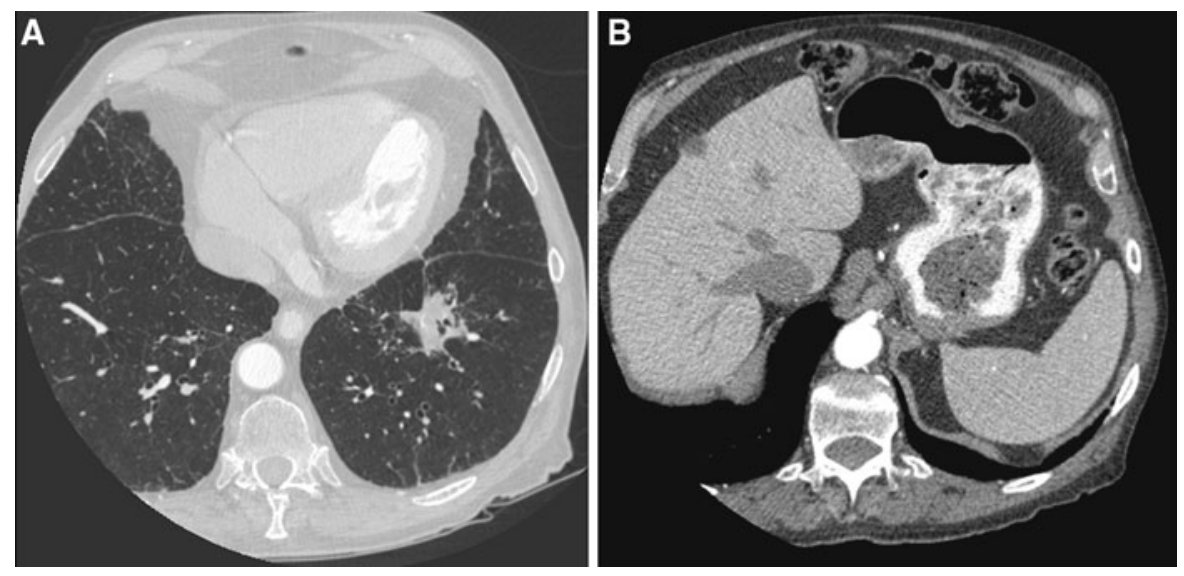

Fig. 4 84-year-old man with AF. a CT-slice at lung window with illustration of pulmonary infiltration of unknown dignity. b CTslice of the upper abdomen with demonstation of multiple liver lesions requiring further evaluation
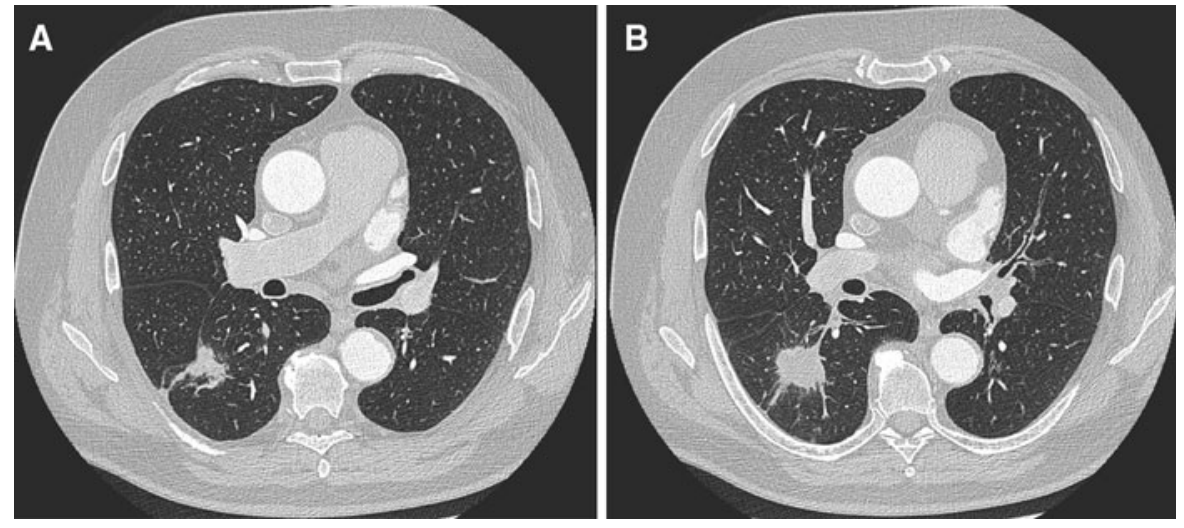

Fig. 5 68-year-old man with AF. a, b Incidental finding for spiculated mass into the right upper lobe, histological proven lung cancer

Table 2 Distribution of clinical non-significant extra cardiac findings (Group 2)

\begin{tabular}{ll}
\hline Clinically non-significant findings & Patients \%/[n/198] \\
\hline Mild degenerative spine disease & $31.5 / 6$ \\
Small and linear lung opacity & $8.5 / 17$ \\
Mediastinal.lymph nodes $<10 \mathrm{~mm}$ & $6 / 12$ \\
Pleural thickening & $5.5 / 11$ \\
Aortic ectasis $<4 \mathrm{~cm}$ & $3 / 6$ \\
Lipoma & $1 / 2$ \\
Pulmonary nodule $<4 \mathrm{~mm}$ & $0.5 / 1$ \\
Steatosis hepatis & $0.5 / 1$ \\
Total & $72 / 113$ \\
\hline
\end{tabular}

contributes to the identification of extracardiac findings. Concerning this matter, the increased cost and additional radiation have to be compared with the clinical need for additional research. To decide whether any incidental or extra cardiac finding should underwent follow up investigations, such as further contrast imaging or clinical correlation, a interdisciplinary determination and justification is definitely fundamental.

The current investigation differs from these earlier EBCT studies in patient population, study methodology, and imaging techniques. Elgin et al. studied a cohort of active military personnel who volunteered for coronary calcium screening [17]. This population was younger and healther than our study population with AF (42 vs. 60.5 years median age) which may explain their lower prevalence of significant and non significant incidental findings. Another reason for the lower incidence of pathological findings might be the different examination mode used for image 
interpretation. As previously decribed by Horton et al. [11], we examined all studies by computer workstation in mediastinal, lung, and bone window settings. Schragin et al. [18] used mediastinal and lung windows printed on hardcopy, and Hundold et al. [12] did not utilize any lung window settings. Moreover, the study design distinctions of EBCT and MDCT are fundamentally different. EBCT does not use a rotating gantry around the patient and therefore achieves high temporal resolution. EBCT coronary calcium scoring also does not require a contrast injection. During MDCT, both a non-contrast enhanced localized image set as well as a contrastenhanced image set can be obtained, increasing the sensitivity for extracardiac findings. Although MDCT with its gantry rotation of $330-420 \mathrm{~ms}$ cannot achieve the temporal resolution of EBCT, its multiple detectors allow for thinner slice thickness and therefore improved spatial resolution. MDCT also employs higher tube current allowing for improved tissue penetration. It is reasonable to conclude that differences in the patient populations studied, image acquisition techniques, and image evaluation account for our higher prevalence of incidental findings dicovered by MDCT scan.

The incidence of extracardiac findings in cardiac studies with MDCT was demonstrated by many publications [11-16]. Schietlinger et al. [16] performed cardiac gated MDCT for the evaluation of the pulmonal vein ostia before AF ablation. Their rates of extra cardiac findings (69 vs. $72 \%$ ) and clinical significant findings (24 vs. $31 \%$ ) were analogous to our experience.

Similarly, the majority of their significant extra cardiac findings were pulmonary (72\%) (Fig. 1). Similarly, Onuma et al. performed cardiac gated MDCT for the evaluation of coronary artery disease and non-blinded interpretation of additional findings. Their rate of extra cardiac findings was $58 \%$ in total and $23 \%$ of significant findings needing additional work-up. The majority of their findings were pulmonary $(72 \%)$, and almost a quarter were pulmonary nodules (Fig. 2). This is not surprising given the majority of their patients had angina, and were older (median age of 66 years) with a high prevalence of smokers (52\%). Cardiac analysis was performed using a FOV defined by the same anatomical landmarks utilized in our study, but they reconstructed an additional image set with a large FOV to include the entire chest. The percentage of findings that came from this additional, larger image set was not reported. The different patient population with higher age and higher proportion of male patients and smokers might account for the slight different prevalence of incidental extra cardiac findings compared with our series. Despite differences in methodology and geography in the three studies, similar clinical results were found.

Usually, radiologists and cardiologists read cardiac MDCT examinations. We recommend a co-reading of a radiologist and cardiologist. Into the four EBCT studies $[11,12,16,17]$ the reported prevalence differs significantly between 7,8 and $20.5 \%$ [11, 17, 18] investigated by two radiologists and 53\% [12] investigated by the co-reading of a cardiologist and a radiologist. This acts in concert with our results and a prevalence of extracardiac findings about $72 \%$. In addition to the heart and coronary arteries, these examinations involve scanning and irradiating parts of the thorax and upper abdomen. A small FOV in cardiac imaging enables optimal resolution of the coronary arteries. However, there are portions of the lungs and the upper abdomen included in the examination that are irradiated but not shown on the small FOV images. Consequently, it is important to review images that are reconstructed with a larger FOV for evaluation of the peripheral chest and upper abdomen. A study by Hong et al. assessed the effects of various sizes of reconstructed FOV on image quality and interpretation and found that the different FOV sizes had a negligible effect on coronary calcium score, lesion size and image noise [19]. Volume analysis of the thorax covered in cardiac CT studies by Haller et al. showed that only $35.5 \%$ of the total chest volume was displayed on the small FOV in typical coronary artery CT settings. With a reconstructed large FOV containing the peripheral lungs, $70.3 \%$ of the total chest volume was displayed [15].

In our study, the highest number of significant extra cardiac findings was found in the lungs (47 findings, Figs. 1, 5). This is explained because approximately $70 \%$ of the total chest volume is imaged in cardiac CT studies and only a very limited section of the upper abdomen and mediastinum were included. Therefore the rate of mediastinal (17 findings), abdominal (7 findings) and other (16 findings) significant findings is lower (Fig. 1). On the other hand the highest rate of non-significant findings was found.

into the "other" group (65 findings) followed by pulmonal (29 findings), mediastinal (18 findings), and 
abdominal findings (1 finding). Most of the other findings were mild or degenerative spine disease which is expected given the median age of the study group (Fig. 1).

An important question is whether the diagnosed "Group 1" findings are really clinically significant. Hunold et al. found that in 1812 EBCT examinations, 3 cases of malignant disease were found [12], and Onuma et al. detected 4 malignancies with MDCT in 503 patients with just 6 months of follow-up [14]. The Early Lung Cancer Action Project (ELCAP) evaluated 1,000 asymptomatic smokers who were at least 60 years old with low dose CT $(140 \mathrm{kV}$ and $40 \mathrm{~mA}$ ) and a $10 \mathrm{~mm}$ slice thickness [20]. They discovered noncalcified pulmonary nodules in $23 \%$ of all scans. Of these 233 scans, $12 \%$ were associated with a malignancy. Importantly, $74 \%$ of these malignancies were not evident on chest radiography. Larger nodules were more likely malignant than smaller lesions.

It is unclear, if the potential additional cost, radiation, and invasive procedures to follow-up these findings will provide patients with a mortality benefit or improved quality of life [21]. Although less than $1 \%$ of sub $5 \mathrm{~mm}$ nodules are malignant, the fear of missing a potential early stage cancer has caused wide variation in follow-up recommendations [20, 21]. On the one hand, in some cases cardiac MDCT will provide a definitive diagnosis in either cardiac or non cardiac cases and therefore facilitate the clinical workup and accelerate treatment. On the other hand, it will produce results that may not be clinically important, but require further examination and costs [22]. As one can see from several types of Group 1, the vast majority of these findings could be managed with minimal stress to the patient and minimal cost to the health-care system. Considering some of the more life-threatening conditions that were incidentally detected and the high radiation exposure that patients receive in MDCT, we believe that the full patient anatomy needs to be analyzed for ethical reasons.

Our study has several limitations. Because of differences in reporting it was difficult to match categories. In the previously discussed EBCT and MDCT studies, the field of view used differs from ours but did not result in a marked increase in additional findings $[11,12,14]$. This study was performed in an academic institution and its applicability to the practice environment is uncertain. Another limitation is that follow-up of these findings is not yet complete. Therefore, their ultimate clinical impact is partly unknown.

Further prospective studies have to evaluate whether the use of a larger field of view for heart scans should be established for routine screening without an increase in radiation dose. A larger scan volume, would require higher doses. It is the question, whether the high prevalence of significant extracardiac findings justify this higher radiation dose. It is conceivable that scanning protocols with different distances to cover and fields of view will be chosen depending on the pathology which might be anticipated in high risk patients, such as additional pulmonary scanning in heavy smokers.

In conclusion, a strategy of co-reading between a team of cardiologists and radiologists is recommendable due to the complexity of these examinations. Otherwise additional training is necessary to enable the recognition of extra cardiac findings [22]. The reader who performs MDCT examinations must recognize these potentially significant findings and establish a diagnosis and categorisation.

Open Access This article is distributed under the terms of the Creative Commons Attribution Noncommercial License which permits any noncommercial use, distribution, and reproduction in any medium, provided the original author(s) and source are credited.

\section{References}

1. Hoffmann U, Moselewski F, Cury RC, Ferencik M, Jang IK, Diaz LJ, Abbara S, Brady TJ, Achenbach S (2004) Predictive value of 16-slice multidetector spiral computed tomography to detect significant obstructive coronary artery disease in patients at high risk for coronary artery disease. Circulation 110:2638-2643

2. Nieman K, Cademartiri F, Lemos PA, Raaijmakers R, Pattynama PA, de Feyter PJ (2002) Reliable noninvasive coronary angiography with fast submillimeter multislice spiral computed tomography. Circulation 106:2051-2054

3. Haberl R, Becker A, Leber A, Knez A, Becker C, Lang C, Brüning R, Reiser M, Steinbeck G (2001) Correlation of coronary calcification and angiographically documented stenosis in patients with suspected coronary artery disease: results on 1, 764 patients. J Am Coll Cardiol 37:451-457

4. Raggi P, Callister TQ, Cooil B, He ZX, Lippolis NJ, Russo DJ, Zelinger A, Mahmarian JJ (2000) Identification of patients at increased risk of first unheralded acute myocardial infarction by electron beam computed tomography. Circulation 101:850-855 
5. Arad Y, Spadaro LA, Goodman K, Newstein D, Guerci AD (2000) Prediction of coronary events with electron beam computed tomography. J Am Coll Cardiol 36:1253-1260

6. Haïssaguerre M, Jaïs P, Shah DC, Takahashi A, Hocini M, Quiniou G, Garrigue S, Le Mouroux A, Le Métayer P, Clémenty J (1998) Spontaneous initiation of atrial fibrillation by ectopic beats originating in the pulmonary veins. N Engl J Med 339:659-666

7. Cappato R, Calkins H, Chen SA, Davies W, Iesaka Y, Kalman J, Kim YH, Klein G, Packer D, Skanes A (2005) Worldwide survey on the methods, efficacy, and safety of catheter ablation for human atrial fibrillation. Circulation 111:1100-1105

8. Verlato R, Turrini P, Baccillieri et al (2005) Variability of pulmonary vein anatomy in patients ablated for atrial fibrillation. Europace 7(s3):s11 Ref Type: Abstract

9. Wood MA, Wittkamp M, Henry D, Martin R, Nixon JV, Shepard RK, Ellenbogen KA (2004) A comparison of pulmonary vein ostial anatomy by computerized tomography, echocardiography, and venography in patients with atrial fibrillation having radiofrequency catheter ablation. Am J Cardiol 93:49-53

10. Jongbloed MR, Dirksen MS, Bax JJ, Boersma E, Geleijns K, Lamb HJ, van der Wall EE, de Roos A, Schalij MJ (2005) Atrial fibrillation: multi-detector row CT of pulmonary vein anatomy prior to radiofrequency catheter ablation-initial experience. Radiology 234:702-709

11. Horton KM, Post WS, Blumenthal RS, Fishman EK (2002) Prevalence of significant noncardiac findings on electronbeam computed tomography coronary artery calcium screening examinations. Circulation 106:532-534

12. Hunold P, Schmermund A, Seibel RM, Grönemeyer DH, Erbel R (2001) Prevalence and clinical significance of accidental findings in electron-beam tomographic scans for coronary artery calcification. Eur Heart J 22:1748-1758

13. MacMahon H, Austin JH, Gamsu G, Herold CJ, Jett JR, Naidich DP, Patz EF Jr, Swensen SJl (2005) Guidelines for management for small pulmonary nodules detected on CT scans: a statement from the Fleischner Society. Radiology 237:395-400
14. Onuma Y, Tanabe K, Nakazawa G, Aoki J, Nakajima H, Ibukuro K, Hara K (2006) Noncardiac findings in cardiac imaging with multidetector computed tomography. J Am Coll Cardiol 48:402-406

15. Haller S, Kaiser C, Buser P, Bongartz G, Bremerich J (2006) Coronary artery imaging with contrast-enhanced MDCT: extracardiac findings. AJR Am J Roentgenol 187:105-110

16. Schietlinger BJ, Bozlar U, Hagspiel KD, Norton PT, Greenbaum HR, Wang H, Isbell DC, Patel RA, Ferguson JD, Gay SB, Kramer CM, Mangrum JM (2008) The prevalence of extracardiac findings by multidetector computed tomography before atrial fibrillation ablation. Am Heart J 155(2):254-259

17. Elgin EE, O'Malley PG, Feuerstein I, Taylor AJ (2002) Frequency and severity of "incidentalomas" encountered during electron beam computed tomography for coronary calcium in middle-aged army personnel. Am J Cardiol 90:543-545

18. Schragin JG, Weissfeld JL, Edmundowicz D, Strollo DC, Fuhrman CR (2004) Non-cardiac findings on coronary electron beam computed tomography scanning. J Thorac Imaging 19:82-86

19. Hong C, Pilgram TK, Zhu F, Bae KT (2004) Coronary artery calcification: effect of size of field of view on multidetector row CT measurements. Radiology 233:281-285

20. Henschke CI, Naidich DP, Yankelevitz DF, McGuinness G, McCauley DI, Smith JP, Libby D, Pasmantier M, Vazquez M, Koizumi J, Flieder D, Altorki N, Miettinen OS (2001) Early lung cancer action project: a summary of the findings on baseline screening. Oncologist 6:147-152

21. Swensen SJ, Jett JR, Hartman TE, Midthun DE, Sloan JA, Sykes AM, Aughenbaugh GL, Clemens MA (2003) Lung cancer screening with CT: Mayo Clinic experience. Radiology 226:756-761

22. Budoff MJ, Fischer H, Gopal A (2006) Incidental findings with cardiac CT evaluation: should we read beyond the heart? Catheter Cardiovasc Interv 68(6):965-973 